\title{
Perceptual Organization of Objects Placed on a Table and Their Level of Disorder
}

\author{
Manabu Gouko \\ Department of Mechanical Engineering and Intelligent Systems, Faculty of Engineering, Tohoku Gakuin \\ University, Tagajo, Japan \\ Email: gouko@mail.tohoku-gakuin.ac.jp
}

Received 19 April 2016; accepted 16 May 2016; published 19 May 2016

Copyright (C) 2016 by author and Scientific Research Publishing Inc.

This work is licensed under the Creative Commons Attribution International License (CC BY). http://creativecommons.org/licenses/by/4.0/

(c) (i) Open Access

\begin{abstract}
In this study, the author investigated the relationship between the layout of objects placed on a table and tidying up. The author developed a system that can tidy up through cooperation between a robot and human. For realizing this system, it was necessary to investigate the types of instances in which people think a table top is disorderly. The author conducted an experiment in which participants were asked to evaluate the disorder condition of presented table images. The participants were also asked to answer how many objects on a table would be collected into groups. The results indicated that objects organized into more groups were rated as untidier than objects organized into fewer groups.
\end{abstract}

\section{Keywords}

Disorder, Table Top, Perceptual Organization

\section{Introduction}

In an aging society, a robot that supports our daily lives is crucial. Above all, housework, cleaning, and tidying up contribute to the realization of a comfortable living environment (Thomas \& Don, 2006). The automatic cleaning robot "Roomba” (iRobot) has grown in popularity. It can move around a room and collect trash. However, an automatic system that can tidy up disordered things has not been developed.

To develop an automatic tidying system, it is necessary to determine the function that judges the necessity of tidying up. In this study, the author focused on tidying up a table top. The author conducted an experiment in which participants were asked to evaluate the order condition of presented table images. Using data on the evaluation of the table condition, the judgement that the table top is ordered or disordered can be calculated automatically. 
This paper is organized as follows. Section 2 describes works related to this study. The experimental setup is described in Section 3. Discussion of the experimental results is presented in Section 4. Conclusions and descriptions of future work are presented in Section 5.

\section{Related Works}

\subsection{Tidying-Up System for a Table Top}

Nishimoto et al. proposed the system Table Cross, which encourages humans to tidy up a table top (Nishimoto et al., 2011). The system observes the degree of disorder of a table located in a shared work space and informs workers via their PC desktop. It automatically generates garbage icons on the desktops of the workers' PCs, reflecting the disorder of the table top. The system calculates a ratio between the occupancy area of the object on a table and the total table top. Nishimoto et al. define this ratio as the degree of disorder of the table: The higher the ratio, the more disordered the table top situation. In this study, the layout of the objects was not considered.

\subsection{Order and Disorder of a Visual Stimulus}

Matsuda and Kaneko investigated the stimulus factors giving rise to impressions of order and disorder (Matsuda $\&$ Kaneko, 2014). In this study, they used visual stimuli comprising $4 \times 4$ square areas and presented them to participants. The participants evaluated the disorder of the shown patterns. The results of this research indicated that a valuing of gathering and local repetition affect impressions of order and disorder.

Studies on the goodness and complexity of presented patterns have been conducted. Garner and Clement investigated the relationship between the goodness of a pattern and uncertainty (Garner \& Clement, 1963). In this research, they used patterns shown in Figure 1. These patterns were presented to participants. Participants then evaluated the goodness of the pattern. The results indicated a tendency in which a participant thinks that a good pattern is one that cannot change easily to reflection or rotation conversion.

Chapman investigated what type of pattern features affect complexity (Chipman, 1977). In his experiment, a two dimensional pattern made by combining black and white squares was adopted. Chapman defined the features of the pattern using quantitative variables and structural variables. For example, the quantitative variable is the number representing the boundary around a black and white square. The result showed that the quantitative variable affects the limit of pattern complexity and that the structural variables reduce complexity.

Kodama and Miura explored the relationship between pattern goodness and perceptual organization (Kodama \& Miura, 2011). Perceptual organization refers to the informational processes structuring visual information into a coherent group. In the experiment, dot patterns were presented to participants. Following this, the goodness of each pattern was evaluated, and participants answered questions regarding a number of groups of perceptual organization. The results showed that there is a tendency for participants who answered by claiming that the dots are collected to hold that the pattern is good.
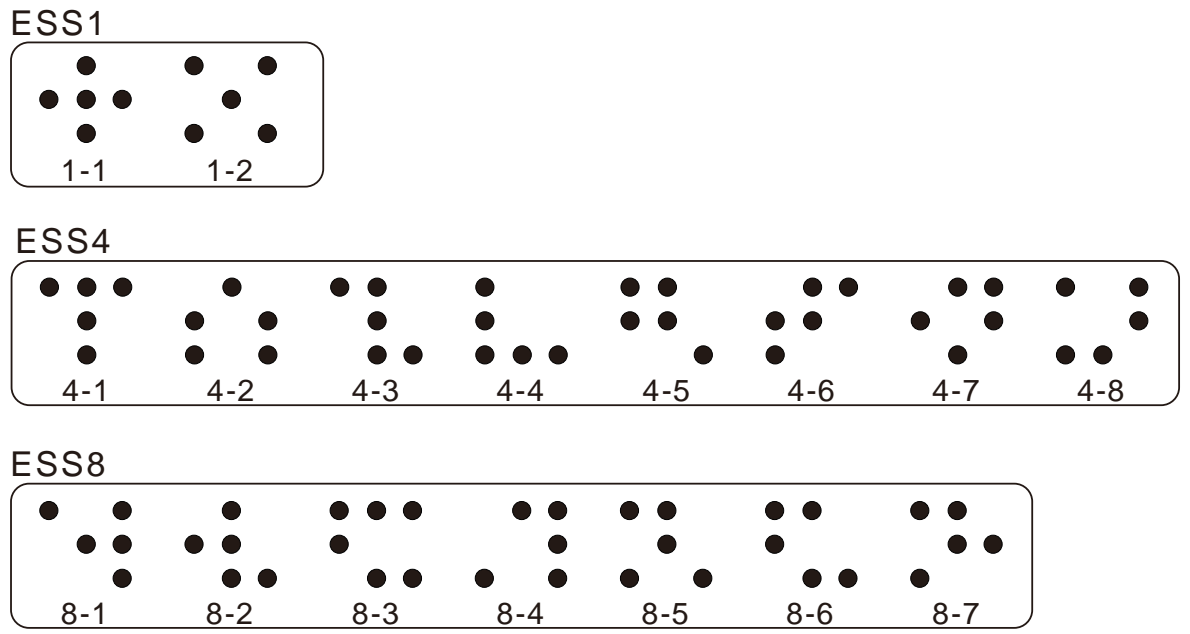

Figure 1. Stimuli used in the study (Garner \& Clement, 1963). 


\subsection{Hypotheses}

Many previous studies have used artificial patterns (e.g., black and white squares and dots) as visual stimuli. However, people hardly see such patterns in everyday life. Therefore, in this study, a visual stimulus pattern made up of images of actual stationery was used. In this research, the author assume the following two hypotheses based on the results of previous researches:

1) There is a tendency for the persons who answer that the stationery on the table has been collected to answer that the table has been tidied up.

2) There is a tendency for persons to answer that the table has been tidied up when the layout of the stationery is difficult to be converted.

The first and second hypotheses are based on previous research (Kodama \& Miura, 2011) and (Garner \& Clement, 1963), respectively.

\section{Experiment}

Experiment participants evaluated the degree of order in presented visual stimulus (patterns). The author used patterns consisting of several images of stationery located on a table. Participants responded into how many groups stationery was classified. In this chapter, the author explain how to make the visual patterns and how to conduct the experimental procedure.

\subsection{Visual Stimulus Pattern}

The visual stimulus pattern was made based on a pattern used in a previous study by Garner and Clement (Garner \& Clement, 1963). These researchers used the pattern shown in Figure 1. These patterns were produced by placing dots in the center of cells of an imaginary 3-by-3 square matrix. The patterns were those that could be generated with five dots, and with the restriction that each row and each column contain at least one dot. Seventeen patterns in Figure 1 were named basic patterns.

The number that set the pattern was produced when it was converted by reflection or rotation. The pattern, with the basic pattern itself, was classified using the numbers 1 , 4, or 8 . The names of the classifications are ESS1, ESS4, and ESS8 (ESS: Equivalence Set Size). Each class includes $2(=2 \times 1), 32(=8 \times 4), 56(=7 \times 8)$ patterns. 32 patterns belonging to ESS4 and 56 patterns belonging to ESS8 were used for producing visual stimuli patterns, named table patterns.

The table pattern used in the experiment explained later was produced by replacing the dots with actual stationery images. Figure 2 shows actual stationery images. Details regarding the actual stationery images used are as follows:

(a) A stapler and two highlighter pens;

(b) Four permanent markers;

(c) A calculator;

(d) Scissors, an eraser, and a mechanical pencil;

(e) A cutter and two stick paste.

Each pattern from (a) to (e) was rotated by a unit of 90 degrees, and four patterns were generated. Figure 3 shows the procedure for making the table pattern. The stationery image, correspondence of the dot, and direction (four) of the stationery images were chosen at random. The table pattern was surrounded by a circle. This circle represents the edge of the table. The thickness of its line is $0.5 \mathrm{~mm}$. Table patterns are 1/10th the size of an 800 $\mathrm{mm}$ diameter table. The sizes of each piece of stationery are also in the ratio of 1/10th.

\subsection{Experimental Procedure}

In the experiment, a total of 88 table patterns were generated from dot patterns belonging to EES4 and EES8. The table patterns were printed on A4 paper and presented to a participant. Four gray-scale printed table patterns were located on one page. The presentation order of the table picture was random for every participant.

Eleven people participated in the experiment (nine males and two females, aged 18 - 23). All participants were Japanese university students and were provided the following instructions before the experiment: "Participants will be shown the image of a table top on which stationery has been placed", "From the point of view of order, the participants will evaluate the presented image", "The participants rated using a 7-level semantic diffe- 
rential scale method (from 1: It is very much tidied up, 7: It is very much in disorder)", and "By drawing a line directly on to the printed paper, participants answer into how many groups stationery is classified". This instruction was presented to participants with a presentation of Figure 4 (example answers).

\section{Results and Discussion}

Figure 5 shows the correlations between the number of organized groups and order rating values. In this figure,

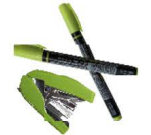

(a)

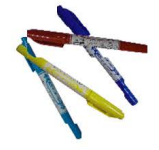

(b)

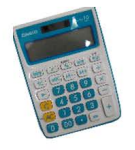

(c)

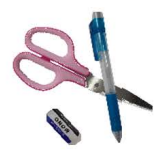

(d)

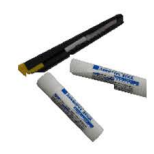

(e)

Figure 2. Stationary images.

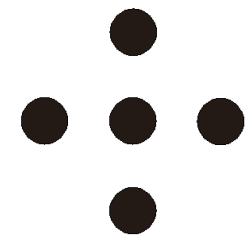

Replacing the dots with actual stationery images

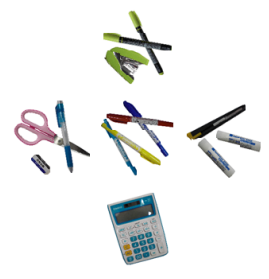

Surrounding the images with a circle.

Figure 3. Procedure of making the table pattern.
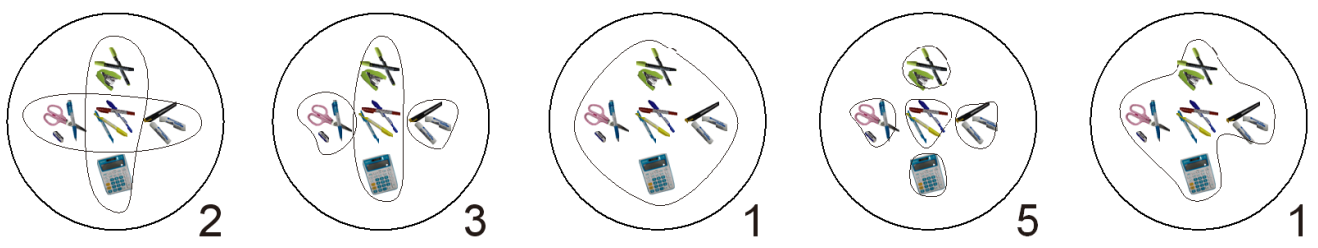

Figure 4. Example answers and the number of organized groups.

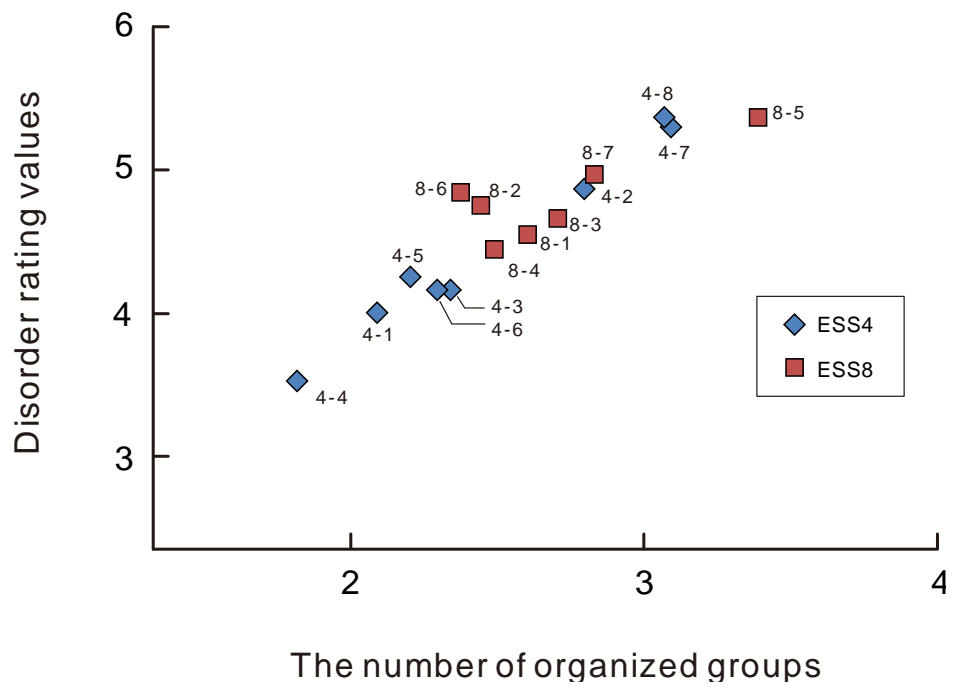

Figure 5. Correlations between the number of organized groups and disorder rating values. 
each mark expresses the average value for every pattern (4-1 - 8-6). The correlation coefficient of the table patterns based on ESS4 is $0.990(p<0.001)$. The correlation coefficient of the table patterns based on ESS8 is $0.802(p<0.05)$. These results support the first hypothesis that there is a tendency for participants who answered that the stationery on the table was collected to answer that the table has been tidied up.

However, the second hypothesis was not established. This hypothesis was that there is a tendency for participants to answer that the table has been tidied up when the layout of the stationery is hard to be converted. If this hypothesis were true, participants would have been expected to answer that the patterns based on EES4 were tidier than those based on EES8. However, the rating values of the patterns based on ESS4-2, 4-7 and 4-8 were low compared with some patterns based on ESS8. This result indicates that the context of the table top affects the participants' cognitions.

\section{Conclusion and Future Work}

In this study, the author investigated the relationship between the layout of objects placed on a table and perspectives on whether they are tidy. The author conducted an experiment in which participants were asked to evaluate the order condition of presented table images. The participants were also asked to specify how many groups the objects on a table would be collected into. The result reveals that things organized into fewer groups are rated as tidier than those organized into more groups. The results indicated that objects organized into more groups were rated as untidier than objects organized into fewer groups.

In this experiment, the author used a visual stimulus that was created by replacing dots with multiple stationery images (Figure 3). However, stationery images include complexity in and of themselves. Therefore, the author will conduct the same experiment using the visual stimulus that was created by replacing the dots with single stationery images. In the future, the author will also conduct an experiment that expands the layout range from ESS4 - ESS8 to ESS1 - ESS16.

\section{Acknowledgements}

This work was supported by JSPS KAKENHI Grant-in-Aid for Scientific Research(C) 15K00363.

\section{References}

Chipman, S. F. (1977). Complexity and Structure in Visual Patterns. Journal of Experimental Psychology, 106, 269-301. http://dx.doi.org/10.1037/0096-3445.106.3.269

Garner, W. R., \& Clement, D. E. (1963). Goodness of Pattern and Pattern Uncertainty. Journal of Verbal Learning \& Verbal Behavior, 2, 446-452. http://dx.doi.org/10.1016/S0022-5371(63)80046-8

Kodama, Y., \& Miura, K. (2011). Pattern Goodness and Perceptual Organization. The Japanese Journal of Psychology, 82, 277-282. (In Japanese). http://dx.doi.org/10.4992/jjpsy.82.277

Matsuda, Y., \& Kaneko, H. (2014). Gathering and Repetition of the Elements in an Image Affect the Perception of Order and Disorder. International Journal of Affective Engineering, 13, 167-173. http://dx.doi.org/10.5057/ijae.13.167

Nishimoto, K., Ikenoue, A., Shimizu, K., Tajima, T., Tanaka, Y., Baba, Y., \& Wang, X. (2011). TableCross: Exuding a Shared Space into Personal Spaces to Encourage Its Voluntary Maintenance (pp. 1423-1428). Proceedings of CHI2011 Extended Abstract. ACM New York. http://dx.doi.org/10.1145/1979742.1979785

Thomas, F., \& Don, T. (2006). 5 S for the Office: Organizing the Workplace to Eliminate Waste. New York: Productivity Press. 